\title{
Highly Stable Passively Q-Switched Erbium-Doped All-Fiber Laser Based on Niobium Diselenide Saturable Absorber
}

\author{
Ping Hu ${ }^{1,2}$, Jiajia Mao ${ }^{1}$, Hongkun Nie ${ }^{1}$, Ruihua Wang ${ }^{1}$, Baitao Zhang ${ }^{1,2,3}$, Tao Li 1,2,4 , Jingliang He $^{1,2,3}$ \\ and Kejian Yang $1,2,3,4,5, *$
}

1 Institute of Novel Semiconductors, State Key Laboratory of Crystal Materials, Shandong University, Jinan 250100, China; 202020452@mail.sdu.edu.cn (P.H.); 201912560@mail.sdu.edu.cn (J.M.); hknie@sdu.edu.cn (H.N.); rhwang@sdu.edu.cn (R.W.); btzhang@sdu.edu.cn (B.Z.); litao@sdu.edu.cn (T.L.); jlhe@sdu.edu.cn (J.H.)

2 China Key Laboratory of Laser \& Infrared System, Ministry of Education Shandong University, Qingdao 266237, China

3 Collaborative Innovation Center of Light Manipulations and Applications, Shandong Normal University, Jinan 250358, China

4 Shenzhen Research Institute, Shandong University, Shenzhen 518057, China

5 State Key Laboratory of Quantum Optics and Quantum Optics Devices, Shanxi University, Taiyuan 030006, China

* Correspondence: k.j.yang@sdu.edu.cn

Citation: Hu, P.; Mao, J.; Nie, H.; Wang, R.; Zhang, B.; Li, T.; He, J.; Yang, K. Highly Stable Passively Q-Switched Erbium-Doped All-Fiber Laser Based on Niobium Diselenide Saturable Absorber. Molecules 2021 26, 4303. https://doi.org/10.3390/ molecules26144303

Academic Editor: Elena Cariati

Received: 11 May 2021

Accepted: 18 June 2021

Published: 16 July 2021

Publisher's Note: MDPI stays neutral with regard to jurisdictional claims in published maps and institutional affiliations.

Copyright: (C) 2021 by the authors. Licensee MDPI, Basel, Switzerland. This article is an open access article distributed under the terms and conditions of the Creative Commons Attribution (CC BY) license (https:/ / creativecommons.org/licenses/by/ $4.0 /)$.

\begin{abstract}
A saturable absorber (SA) based on niobium diselenide $\left(\mathrm{NbSe}_{2}\right)$, which is a layered transition metal dichalcogenide (TMD) in the VB group, is fabricated by the optically driven deposition method, and the related nonlinear optical properties are characterized. The modulation depth, saturable intensity, and nonsaturable loss of the as-prepared $\mathrm{NbSe}_{2}$ nanosheet-based SA are measured to be $16.2 \%, 0.76 \mathrm{MW} / \mathrm{cm}^{2}$, and $14 \%$, respectively. By using the as-fabricated $\mathrm{NbSe}_{2} \mathrm{SA}$, a highly stable, passively Q-switched, erbium-doped, all-fiber laser is realized. The obtained shortest pulse width is $1.49 \mu \mathrm{s}$, with a pulse energy of $48.33 \mathrm{~nJ}$ at a center wavelength of $1560.38 \mathrm{~nm}$. As far as we know, this is the shortest pulse duration ever obtained by an $\mathrm{NbSe}_{2} \mathrm{SA}$ in a Q-switched fiber laser.
\end{abstract}

Keywords: niobium diselenide; fiber laser; Q-switched; saturable absorber

\section{Introduction}

Short-pulsed, all-fiber lasers have been widely used in various fields such as material processing, medical diagnosis, industrial processing, light detection, and ranging (LIDAR, also LADER), optical fiber communication, and spectroscopy [1-3]. The emergence and development of Q-switching technology is an important breakthrough in the history of laser development [4]. It compresses the laser energy into extremely narrow pulses for emission, so that the peak power of the light source can be increased by several orders of magnitude. The Q-switching mechanism mainly involves the generation of short pulses, whose pulse duration can reach the order of microseconds ( $\mu s)$, nanoseconds (ns), and even picoseconds (ps), while the repetition frequency can range from several $\mathrm{Hz}$ to hundreds of kilohertz $(\mathrm{kHz})[5,6]$. Passive Q-switching technology based on SAs has been a popular way to achieve pulsed lasers due to its advantages of compactness, high efficiency, and low cost [7-9]. In recent years, with the development of nonlinear optical materials, twodimensional (2D) materials have received widespread attention due to their novel electronic and optical performance [10-13]. Despite the thin atomic layer, many 2D materials interact strongly with light in a broad spectral band, which is helpful for optical modulation. However, the nonlinear optical response characteristics of the 2D materials inevitably affect the repeatability and reliability of pulsed fiber lasers [14]. Therefore, exploring suitable materials with excellent nonlinear optical response properties is still the key to the development of high-performance pulsed fiber lasers. Among the layered 2D materials, 
TMDs have been widely studied due to their excellent nonlinear optical performance introduced by the high third-order nonlinear susceptibility [15]. Compared with other typical 2D materials, such as graphene and black phosphorous (BP), the band gap of TMDs increases with the decrease in the number of layers [16], which introduces broadband absorption helpful for optical modulation [17]. In addition, TMDs have high carrier mobility [18], which makes them possess fast nonlinear response to incident light, thus making them suitable for generating narrow pulses.

Among the TMD materials, extensive research has been conducted on materials with semiconductor properties such as $\mathrm{WS}_{2}, \mathrm{MoS}_{2}, \mathrm{WSe}_{2}$, and $\mathrm{MoSe}_{2}$ [19-22], which show promising performance in generating pulsed laser pulses. Compared with the TMD materials with semiconductor properties, 2D nanostructured TMDs with metal layers, such as $\mathrm{NbS}_{2}$ and $\mathrm{NbSe}_{2}$, exhibit lower fabrication cost, stronger optical absorption, and higher carrier mobility [23-26]. Recently, $\mathrm{NbSe}_{2}$ has been clearly proven to have good saturable absorption properties in the spectral range from 1 to $1.5 \mu \mathrm{m}$, due to ultra-fast saturation recovery time and wide absorption band [27-30]. Furthermore, $\mathrm{NbSe}_{2}$ has excellent environmental stability, so it is very helpful for generating long-term stable laser pulses, especially in fiber lasers. In 2018, Shi et al. prepared an $\mathrm{NbSe}_{2}$ quantum $\operatorname{dot}(\mathrm{QD})$-based SA with modulation depth and saturable absorption intensity of $3.72 \%$ and $3.155 \mathrm{GW} / \mathrm{cm}^{2}$, respectively, and employed it to achieve a Q-switching fiber laser, which delivered pulses with a duration of $2.53 \mu$ s and single pulse energy of $98.19 \mathrm{~nJ}$ at a center wavelength of $1533 \mathrm{~nm}[27,28]$. The SA was fabricated by dropping $\mathrm{NbSe}_{2}$ QDs solution onto the side-projection face of a D-shaped fiber, which could improve both the damage threshold and thermal stability of the SA. Although $\mathrm{NbSe}_{2} \mathrm{QDs}$ and D-shaped fiber have stronger evanescent field interactions than the ordinary single-mode fiber, greater coupling loss would also be introduced during the fusion splicing process with single-mode fiber. In addition, the special structure of the SA based on D-shaped fiber also determines relatively low modulation depth and high saturable absorption intensity, which is harmful for achieving a low-threshold Q-switched laser with narrow pulse duration.

In this paper, a kind of $\mathrm{NbSe}_{2}$ nanosheet $\mathrm{SA}$ is fabricated by the optically driven deposition method, and the nonlinear saturable absorption properties are characterized. The $\mathrm{NbSe}_{2}$ nanosheet-based SA shows a large modulation depth of $16.2 \%$, and a low saturation intensity of $0.76 \mathrm{MW} / \mathrm{cm}^{2}$, which provides the possibility to achieve narrow laser pulses with low threshold. By employing the as-prepared $\mathrm{NbSe}_{2} \mathrm{SA}$ in an all-fiber, erbium-doped fiber laser, a stable passive Q-switching operation is realized. The obtained pulse repetition rate ranges from 15.12 to $64.14 \mathrm{kHz}$ within the pump power range. The shortest pulse duration is $1.49 \mu \mathrm{s}$, corresponding to a single pulse energy of $48.33 \mathrm{~nJ}$. As far as we know, this is the shortest pulse duration ever obtained by an $\mathrm{NbSe}_{2} \mathrm{SA}$ in a Q-switched fiber laser so far. The results clearly show that such a kind of $\mathrm{NbSe}_{2} \mathrm{SA}_{\text {as }}$ great potential for achieving all-fiber pulsed laser operation.

\section{Preparation and Characterization of $\mathrm{NbSe}_{2}$}

\subsection{Preparation of $\mathrm{NbSe}_{2}$}

The $\mathrm{NbSe}_{2}$ nanosheet SA is prepared by the optically driven deposition method due to its simplicity and high efficiency. The preparation process is shown in Figure 1. The $\mathrm{NbSe}_{2}$ nanosheet powder was purchased from Shenzhen HAOLAI Technology Co., Ltd., Shenzhen, China. In the preparation method, $10 \mathrm{mg}$ of $\mathrm{NbSe}_{2}$ powder and $10 \mathrm{~mL}$ ethanol are first mixed to form the dispersion of $\mathrm{NbSe}_{2}$ nanosheets. The dispersion is fully stirred for $15 \mathrm{~min}$ and subjected to ultrasonication for $2 \mathrm{~h}$. After that, the dispersion is centrifuged at $3000 \mathrm{rpm}$ for $30 \mathrm{~min}$, until the $\mathrm{NbSe}_{2}$ nanosheets are completely dispersed. Then, $280 \mathrm{mg}$ of polyvinyl acetate (PVA) powder is added into $7 \mathrm{~mL}$ of $\mathrm{NbSe}_{2}$ nanosheet dispersion, and sonicated for $30 \mathrm{~min}$ to prepare a uniform $\mathrm{NbSe}_{2}$-PVA composite with embedded nanosheets. In this special polymer matrix, PVA can prevent oxidation while forming a $\mathrm{NbSe}_{2}$ nanosheet film. Next, the end face of an optical fiber connector is immersed into the prepared $\mathrm{NbSe}_{2}-\mathrm{PVA}$ composite solution. Then, the coated fiber is connected to a laser 
$(976 \mathrm{~nm}, 25 \mathrm{~mW})$ for $20 \mathrm{~min}$. Finally, the coated fiber is disconnected and allowed to dry for $24 \mathrm{~h}$ at room temperature.

(a)

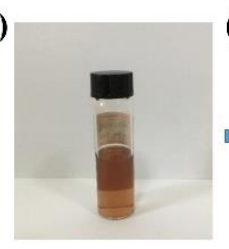

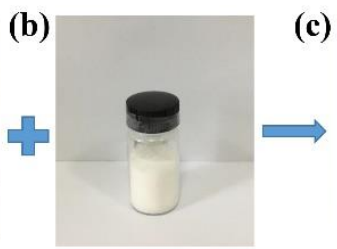

(c)

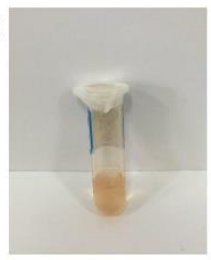

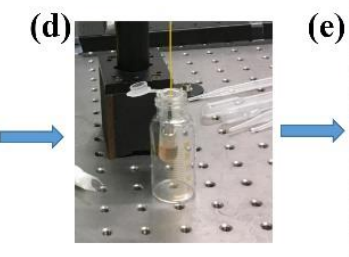

(e)

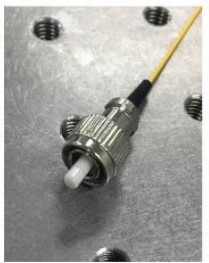

Figure 1. Preparation process of $\mathrm{NbSe}_{2}$-PVA SA. (a) $\mathrm{NbSe}_{2}$ powder; (b) PVA powder; (c) $\mathrm{NbSe}_{2}$-PVA composite dispersion; (d) optically driven deposition setup; (e) $\mathrm{NbSe}_{2}$ nanosheet-coated fiber connector.

\subsection{Characterization of $\mathrm{NbSe}_{2} \mathrm{SA}$ Properties}

The microstructure and thickness of $\mathrm{NbSe}_{2}$ nanosheets are characterized by a transmission electron microscope (TEM) (JEOL, JEM 2100F), which is driven at an accelerating voltage of $200 \mathrm{kV}$. A TEM image of the $\mathrm{NbSe}_{2}$ nanosheet SA with a resolution of $200 \mathrm{~nm}$ is shown in Figure 2. It can be seen that the $\mathrm{NbSe}_{2}$ nanosheets demonstrate clear layer structure and rod shape. The inset is a high-resolution TEM (HRTEM) image of the $\mathrm{NbSe}_{2}$ nanosheets, from which no obvious defects are found, clearly proving the excellent structure of the $\mathrm{NbSe}_{2}$ nanosheets. The lattice distance is about $0.19 \mathrm{~nm}$, which corresponds to the $(1,0,5)$ lattice plane.

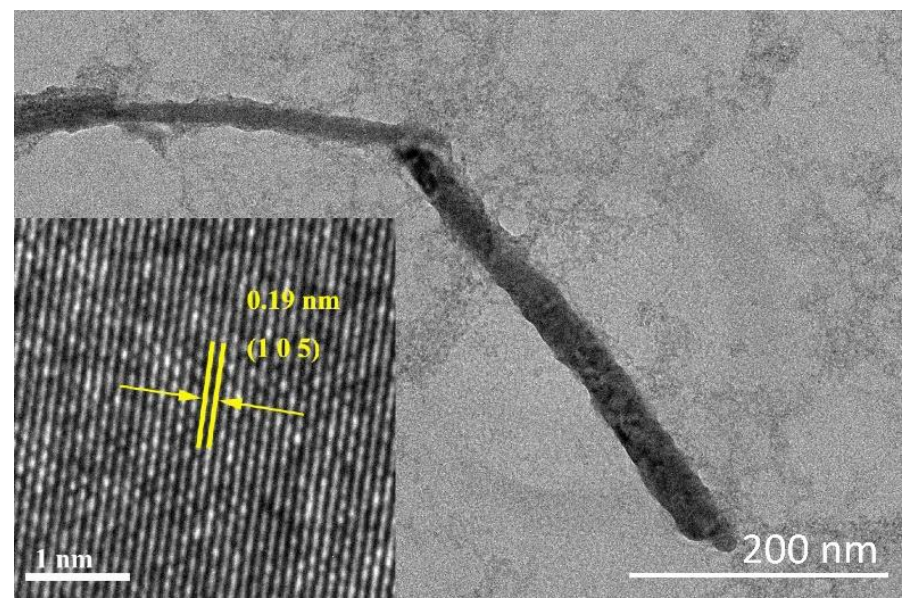

Figure 2. TEM image of $\mathrm{NbSe}_{2}$ nanosheets. Inset: HRTEM image of $\mathrm{NbSe}_{2}$ nanosheets.

In order to confirm the thickness of $\mathrm{NbSe}_{2}$ nanosheets in $\mathrm{NbSe}_{2}-\mathrm{PVA}$ film, atomic force microscopy (AFM) (Dimension Icon, Veeco Instruments Inc., Plainview, NY, USA) measurement is performed. The $\mathrm{NbSe}_{2}$-PVA composite dispersion is deposited onto a quartz substrate by the spin coating method, and dried to form a $\mathrm{NbSe}_{2}-\mathrm{PVA}$ film. To avoid affecting the number of $\mathrm{NbSe}_{2}$ nanosheet layers, the prepared sample is placed in ethanol solvent and sonicated for $10 \mathrm{~min}$ to remove excessive PVA on the surface. Subsequently, the sample is dried for characterization. The corresponding AFM images and height profiles are shown in Figure 3a,b. Three section parts in the scope of Figure $3 a$ are selected to characterize the heights, denoted as section 1 , section 2 , and section 3 , respectively. It can be seen from Figure $3 \mathrm{~b}$ that the thicknesses of the selected $\mathrm{NbSe}_{2}$ nanosheet parts range from 7 to $14 \mathrm{~nm}$. Considering a single-layer $\mathrm{NbSe}_{2}$ nanosheet thickness of about $1.1 \mathrm{~nm}$, the prepared $\mathrm{NbSe}_{2} \mathrm{SA}$ nanosheets are determined to be about 6-13 layers [31]. The few-layered nature of the $\mathrm{NbSe}_{2} \mathrm{SA}$ nanosheets makes them suitable for working at a wavelength of around $1550 \mathrm{~nm}$. 
(a)

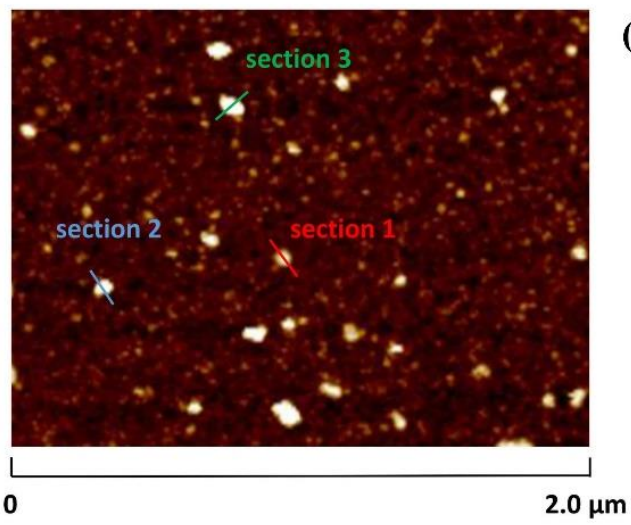

(b)

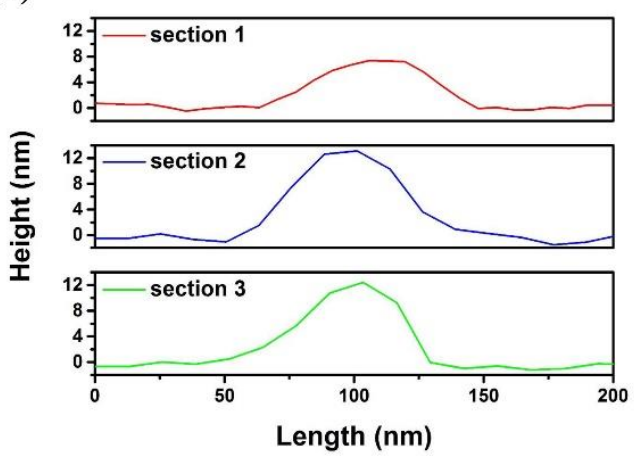

Figure 3. (a) AFM image of $\mathrm{NbSe}_{2} \mathrm{SA}$; (b) height profiles of $\mathrm{NbSe}_{2} \mathrm{SA}$.

A UV/VIS/NIR spectrophotometer (U-3500, Hitachi, Japan) is further used to measure the linear transmission spectrum of $\mathrm{NbSe}_{2}$ nanosheet $\mathrm{SA}$ in a spectral range of 200-1800 nm. As shown in Figure 4, the blue line represents the transmittance of the blank quartz substrate, and the red line represents the transmittance of the $\mathrm{NbSe}_{2}$ nanosheet $\mathrm{SA}$ on the quartz substrate under the same conditions. In the near-infrared spectral range from 900 to $1800 \mathrm{~nm}$, the transmittances of the quartz substrate with the $\mathrm{NbSe}_{2}$ nanosheet $\mathrm{SA}$ and the blank quartz substrate are found to be $87.5 \pm 5.5 \%$ and $92.7 \pm 0.5 \%$, respectively, which shows that the $\mathrm{NbSe}_{2}$ nanosheet SA introduces an absorbance of about $5.2 \%$, indicating the broad absorption properties of $\mathrm{NbSe}_{2} \mathrm{SA}$.

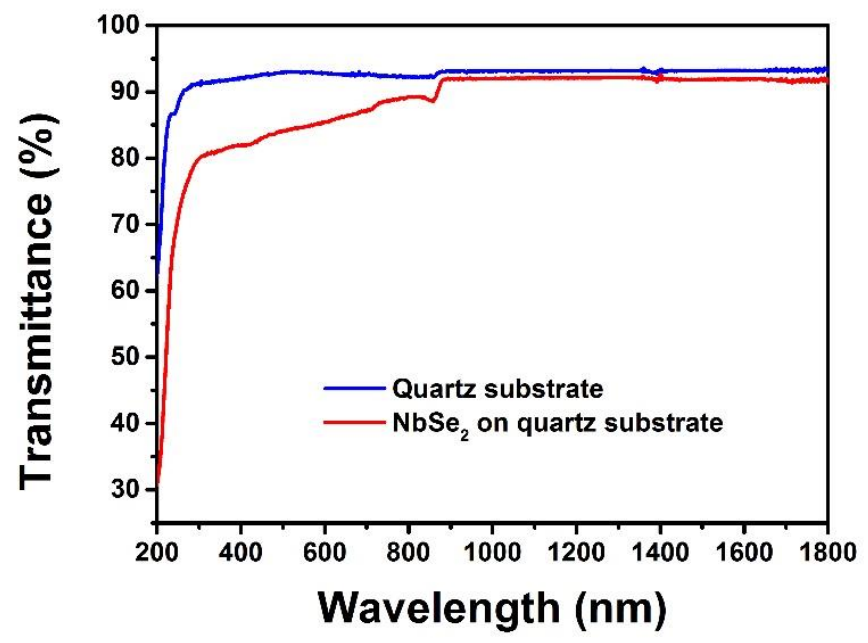

Figure 4. Transmittance of the quartz substrate and $\mathrm{NbSe}_{2}$ based on quartz substrate.

The nonlinear saturable absorption properties of $\mathrm{NbSe}_{2} \mathrm{SA}$ are studied by the balanced dual-detector measurement method, as shown in Figure 5. A femtosecond/picosecond laser is used as a pumping source. The working wavelength of the fiber laser used is $1550 \mathrm{~nm}$, and the fundamental frequency is $10 \mathrm{~Hz}$. The power is changed using a variable optical attenuator (VOA). A typical fiber coupler (50:50) splits the power between the reference and measurement arm. Then, by comparing the values of the two power meters, the transmittance curve of the sample to the laser pulse at different optical powers can be drawn. In the end, the nonlinear, power-dependent, normalized saturation absorption curve based on $\mathrm{NbSe}_{2} \mathrm{SA}$ is shown in Figure 6. It can be seen from the figure that the blue dots represent experimental data. The red line in the figure shows the curve of the saturated absorption formula [32]:

$$
\mathrm{T}(\mathrm{I})=1-\mathrm{Tns}-\Delta \mathrm{T} \times \exp (-\mathrm{I} / \text { Isat })
$$


where $\mathrm{T}(\mathrm{I})$ is the measured transmittance, Tns is the nonsaturable absorbance, $\Delta T$ is modulation depth, $I$ is input laser intensity, and Isat is saturation intensity. By simulating the measured data with Equation (1), the modulation depth, saturation intensity, and nonsaturable loss are $16.2 \%, 0.76 \mathrm{MW} / \mathrm{cm}^{2}$, and $14 \%$, respectively. Compared with the results reported in previous work [33,34], the $\mathrm{NbSe}_{2} \mathrm{SA}$ has a higher modulation depth and a lower saturation intensity in this work. For the D-shaped fiber-based SA, its saturable absorption arises from the interaction between the evanescent field outside the D-shaped fiber and SA, introducing a large unsaturable loss due to the large interaction area. Here, due to the direct light-matter interaction between the $\mathrm{NbSe}_{2}$ and light at the end face of $\mathrm{SMF}$, low unsaturable loss, large modulation depth, and low saturation intensity are easily obtained. The large modulation depth would help generate short pulses [35], while the low saturable intensity makes low-threshold and high-repetition rate Q-switching operation expected. As can be seen from Figure 6, when the laser power gradually increases, the optical transmittance increases to approximately $86 \%$ and remains saturated. $\mathrm{The}_{\mathrm{NbSe}}$ nanosheet sample exhibits a typical saturated absorption curve.

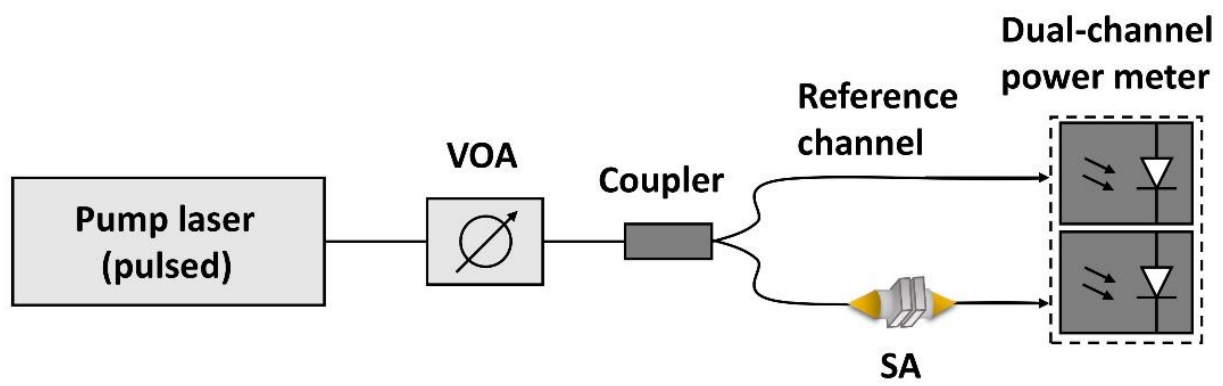

Figure 5. Diagram of experimental setup for balanced dual-detector measurement method.

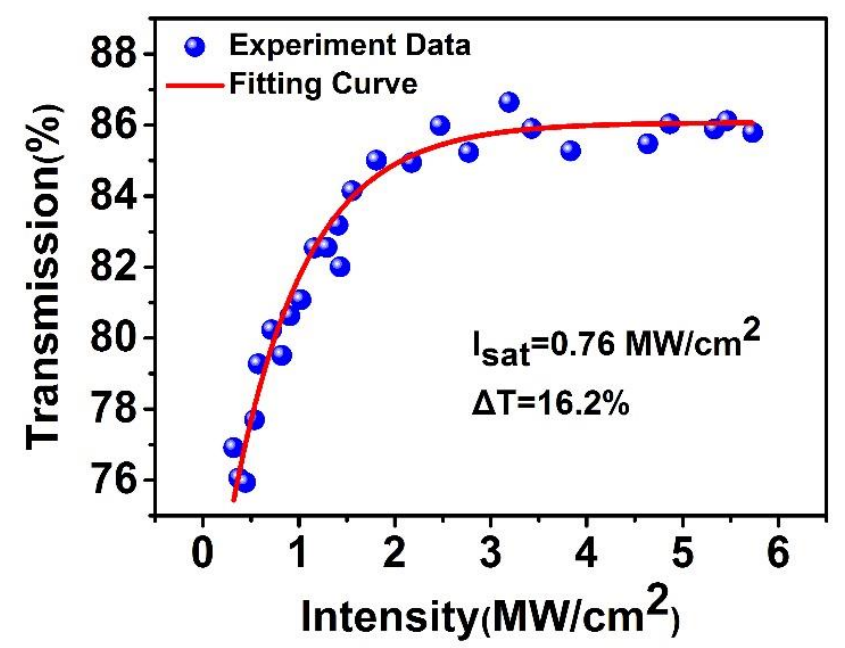

Figure 6. The nonlinear optical absorption properties of the $\mathrm{NbSe}_{2} \mathrm{SA}$.

\section{Experimental Results of Optical Modulation and Discussions}

The experimental setup of the passively Q-switched, erbium-doped fiber laser based on the as-prepared $\mathrm{NbSe}_{2} \mathrm{SA}$ is shown in Figure 7. The ring laser cavity includes pump source, 980/1550 nm wavelength division multiplexer (WDM), polarization controller (PC), 20/80 output coupler (OC), polarization independent isolator (PI-ISO), erbium-doped fiber (EDF), $\mathrm{NbSe}_{2} \mathrm{SA}$, and single-mode fiber (SMF). Among them, the pump source is a $976 \mathrm{~nm}$ laser diode (LD) with a maximum power of $515 \mathrm{~mW}$. The pump light is coupled into the laser cavity through the $980 / 1550 \mathrm{~nm}$ WDM. A $6 \mathrm{~m}$ single mode erbium-doped fiber (Nufern-EDFC-980-HP) is used as the gain medium. The PI-ISO is used to ensure the unidirectional light propagation in the laser cavity. Since the cavity is composed of 
standard SMFs, a PC is required to adjust the polarization state. In addition, an OC with a coupling rate of $20 \%$ is employed to output the laser pulses. The total length of the ring laser cavity is about $11 \mathrm{~m}$. The spectral and temporal properties of the output pulses are characterized by a spectrum analyzer (YOKOGAWA AQ6370B) and a digital oscilloscope (Tektronix DP04104), respectively. The average output power is measured by a power meter (Molectron PM3).

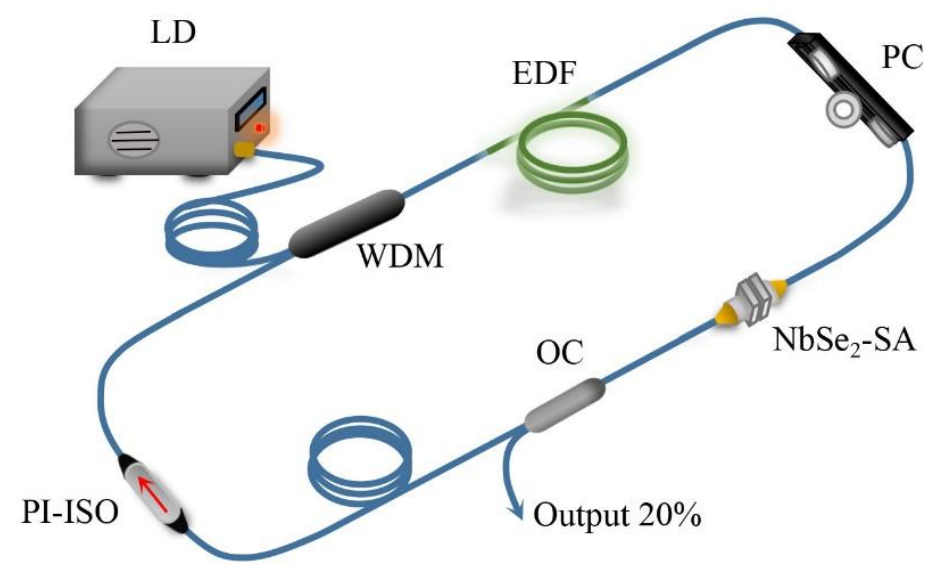

Figure 7. Schematic diagram of the $\mathrm{NbSe}_{2} \mathrm{SA}$ passively Q-switched EDF laser experiment.

During the experiment, the PC is adjusted to change the polarization state of the cavity mode while increasing the pump power, and then the laser output pulses are observed using the spectrum analyzer and digital oscilloscope. When the pump power is increased to $41 \mathrm{~mW}$, the laser begins to work in the Q-switching regime. In order to verify the function of the $\mathrm{NbSe}_{2} \mathrm{SA}$ in the Q-switching, erbium-doped fiber laser, the SA is taken out of the cavity and the cavity length is kept unchanged. By adjusting the PC and pump power carefully, no Q-switching phenomenon is observed. The results affirm that the as-prepared $\mathrm{NbSe}_{2} \mathrm{SA}$ is mainly responsible for the Q-switching operation.

Figure 8 presents the output pulse sequences at different pump powers. The pulses have identical amplitudes even under a pump power above $500 \mathrm{~mW}$, indicating stable Q-switching operations under different pump powers. When the pump power is increased to the maximum of $515 \mathrm{~mW}$, the Q-switched pulses still remain highly stable. Due to the limitation of the pump power available in our laboratory, it is impossible to measure the affordable pump power limit of the $\mathrm{NbSe}_{2} \mathrm{SA}$; however, no damage is observed within the pump power range. In addition, the $\mathrm{NbSe}_{2}$ nanosheet $\mathrm{SA}$ exhibits high long-term stability and oxidation resistance under environmental conditions, which makes the laser remain in stable operation for more than one month, and makes it quite suitable for application in practical optical devices.

Figure 9a presents the temporal pulse shape with the shortest pulse width of $1.49 \mu \mathrm{s}$ recorded under the maximum pump power of $515 \mathrm{~mW}$, corresponding to a repetition rate of $64.14 \mathrm{kHz}$. Figure $9 \mathrm{~b}$ shows the spectrum of the output pulse with a center wavelength at $1560.38 \mathrm{~nm}$. The recorded pulse durations and repetition rates under different pump powers are shown in Figure 9c. When the pump power is increased from 41 to $515 \mathrm{~mW}$, the pulse duration decreases from 13.18 to $1.49 \mu$ s, while the repetition rate increases from 15.12 to $64.14 \mathrm{kHz}$. Figure $9 \mathrm{~d}$ depicts the dependence of output power and single pulse energy on the pump power. Under the maximum pump power of $515 \mathrm{~mW}$, a maximum average output power of $3.1 \mathrm{~mW}$ is obtained with a pulse energy of $48.33 \mathrm{~nJ}$, corresponding to an optical-optical conversion efficiency and slope efficiency of $0.6 \%$ and $0.64 \%$, respectively. The slope efficiency is relatively low due to the high insertion loss of SA, but it is possible to further improve it by optimizing the unsaturated loss of $\mathrm{NbSe}_{2} \mathrm{SA}$ and splitting ratio of OC. 


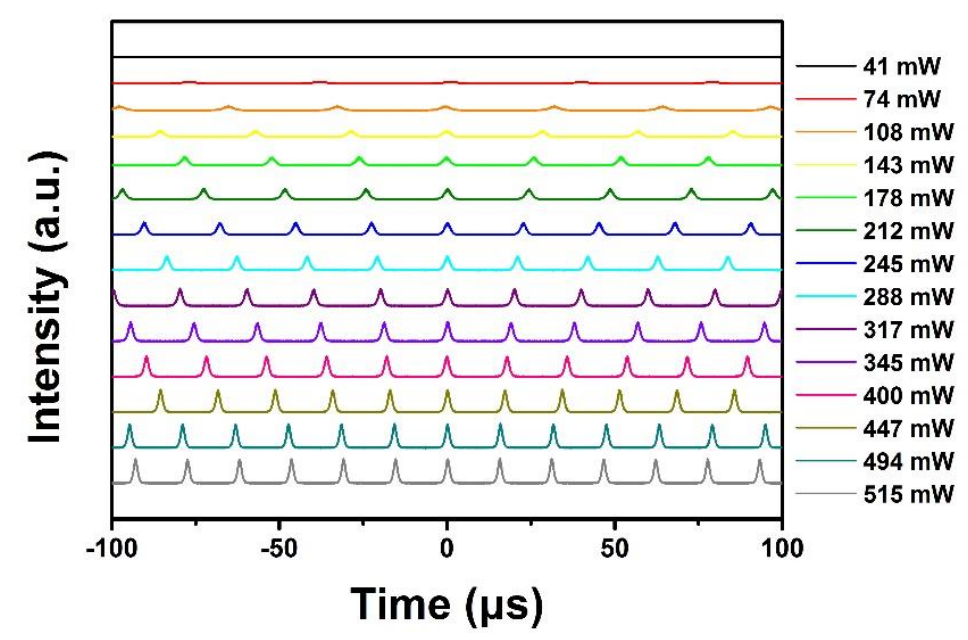

Figure 8. Temporal pulse sequences under different pump powers.
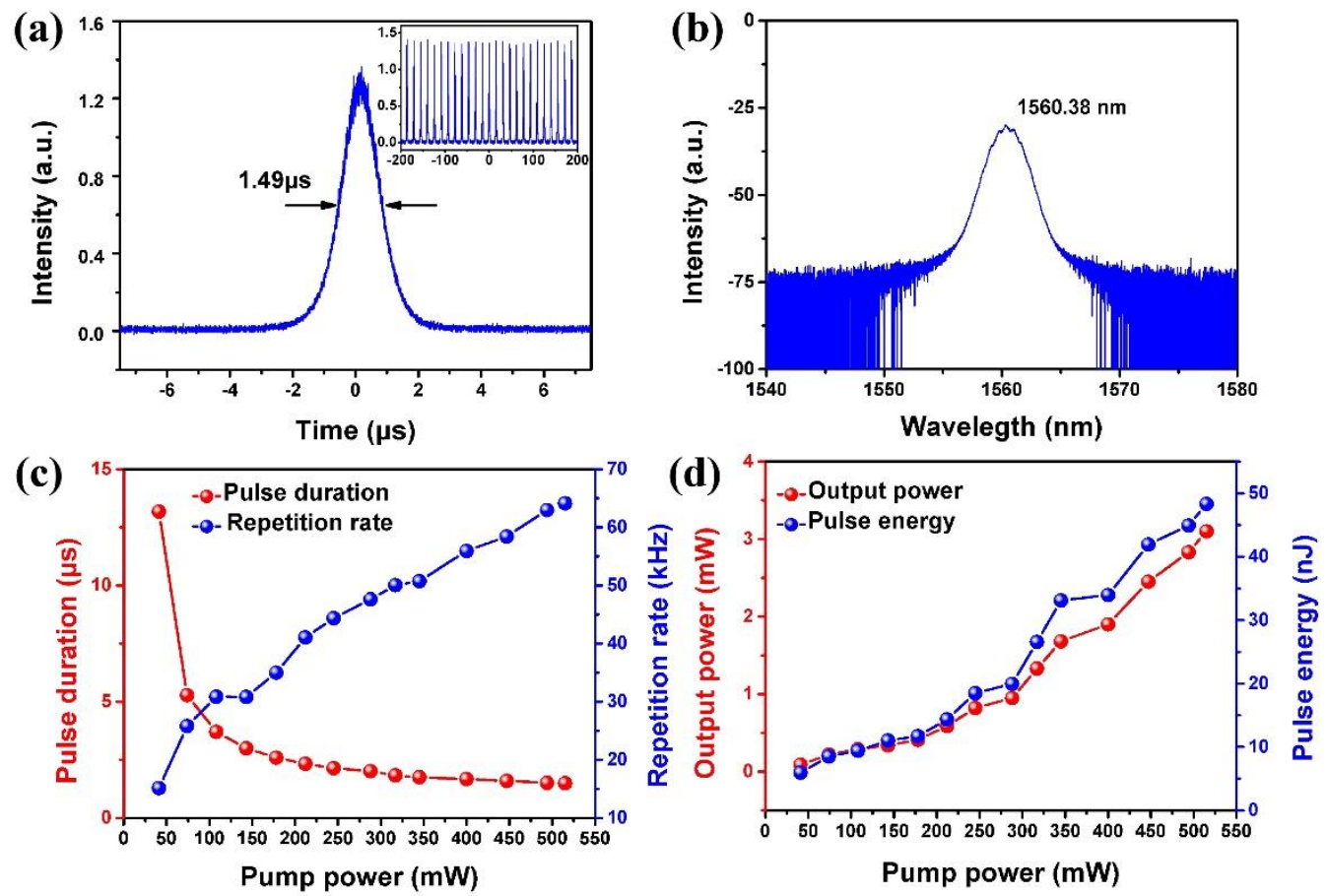

Figure 9. The output pulse characteristics of the $\mathrm{NbSe}_{2} \mathrm{SA}$, passively Q-switched fiber laser. (a) Temporal pulse shape recorded under the pump power of $515 \mathrm{~mW}$, and the inset is the corresponding pulse sequence; (b) laser spectrum recorded under the maximum pump power; (c) pulse durations and pulse repetition rates versus the pump powers; (d) output powers and pulse energies versus the pump powers.

In order to test the long-term stability of the Q-switched fiber laser based on $\mathrm{NbSe}_{2}$ $\mathrm{SA}$, the output spectrum under the maximum pump power is continuously monitored for more than $2 \mathrm{~h}$ with intervals of $30 \mathrm{~min}$. As shown in Figure 10, the output spectrum does not change within $2 \mathrm{~h}$, which proves that the Q-switched, erbium-doped fiber laser has excellent stability at room temperature. The results clearly indicate the as-prepared $\mathrm{NbSe}_{2}$ SA meets the requirements of long-term and stable laser operation under atmospheric conditions, which is very suitable for a nonlinear photonic device to generate narrow output pulses in fiber lasers. 


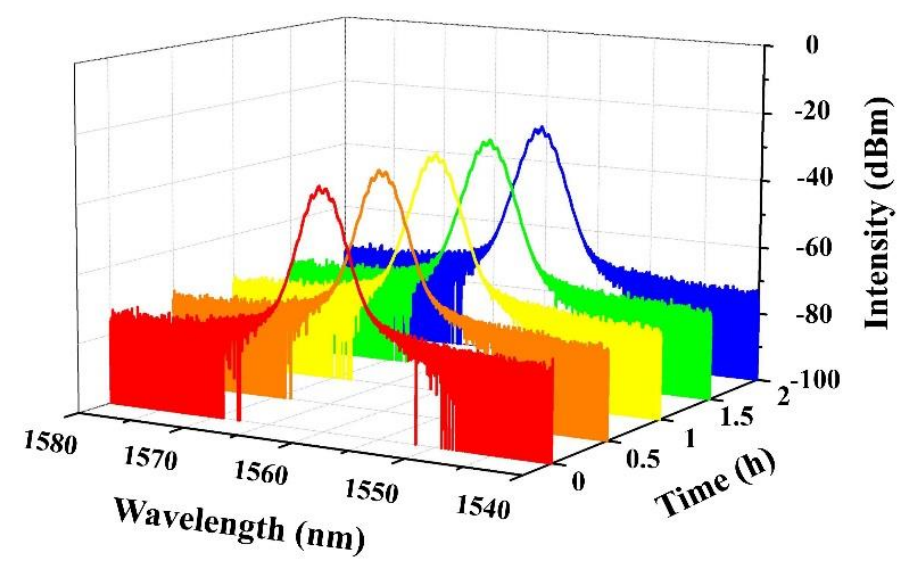

Figure 10. Long-term performance of the $\mathrm{NbSe}_{2} \mathrm{SA} \mathrm{Q}$-switched fiber laser within $2 \mathrm{~h}$ with an interval of $30 \mathrm{~min}$.

For comparison, the performance of passively Q-switched, erbium-doped fiber lasers using different nanomaterial SAs is summarized in Table 1. Compared with other SAs, the $\mathrm{NbSe}_{2}$-based SA generates the shortest pulse duration in this work. It is indicated that the optically driven deposition method is helpful to reduce the unexpected splicing loss of the prepared $\mathrm{NbSe}_{2} \mathrm{SA}$ while retaining high modulation depth and low saturation intensity, which is beneficial for generating short pulses with low pump thresholds.

Table 1. Comparison of passively Q-switched, Er-doped fiber lasers based on different SAs.

\begin{tabular}{cccccc}
\hline SA & $\begin{array}{c}\text { Wavelength } \\
(\mathbf{n m})\end{array}$ & $\begin{array}{c}\text { Pulse Width } \\
(\boldsymbol{\mu} \mathbf{s})\end{array}$ & $\begin{array}{c}\text { Pulse Energy } \\
(\mathbf{n J})\end{array}$ & $\begin{array}{c}\text { Max Output } \\
\text { Power (mW) }\end{array}$ & Refs. \\
\hline graphene & 1539.6 & 3.89 & 28.7 & 3.38 & {$[33]$} \\
$\mathrm{MoS}_{2}$ & 1560 & 3.97 & 131.52 & 6 & {$[34]$} \\
$\mathrm{WS}_{2}$ & 1560 & 3.71 & 126.96 & 4.82 & {$[34]$} \\
$\mathrm{ReS}_{2}$ & 1532 & 2.57 & 38 & 2.48 & {$[36]$} \\
$\mathrm{BP}$ & 1564.16 & 2.98 & 283.91 & 8.55 & {$[37]$} \\
antimonene & 1559.63 & 1.58 & 37.9 & 2.85 & {$[38]$} \\
$\mathrm{NbSe}_{2} \mathrm{QD}$ & 1533 & 2.53 & 98.19 & 2.00 & {$[28]$} \\
$\mathrm{NbSe}_{2}$ & 1560.38 & 1.49 & 48.33 & 3.1 & This work \\
\hline
\end{tabular}

\section{Conclusions}

In conclusion, a high long-term stability, passively Q-switched, erbium-doped allfiber laser is realized by using a few-layered $\mathrm{NbSe}_{2} \mathrm{SA}$ fabricated by the optically driven deposition method. The as-prepared $\mathrm{NbSe}_{2}$ nanosheet $\mathrm{SA}$ shows good homogeneity and uniform thickness. The nonlinear optical properties of the $\mathrm{NbSe}_{2} \mathrm{SA}$ are characterized in the $1550 \mathrm{~nm}$ spectral region, and a large modulation depth of $12.6 \%$ and a low saturation intensity of $0.76 \mathrm{MW} / \mathrm{cm}^{2}$ are demonstrated. By using the as-prepared $\mathrm{NbSe}_{2} \mathrm{SA}$, highly stable Q-switching operation is realized in the erbium-doped all-fiber laser. When the pump power is $515 \mathrm{~mW}$, a maximum average output power of $3.1 \mathrm{~mW}$ is obtained, corresponding to a pulse energy of $48.33 \mathrm{~nJ}$ and a pulse duration of $1.49 \mu \mathrm{s}$. This is the shortest pulse duration ever achieved using an $\mathrm{NbSe}_{2} \mathrm{SA}$ in a passively Q-switched fiber laser, to the best of our knowledge. The results indicate that the $\mathrm{NbSe}_{2} \mathrm{SA}$ is a promising practical photonic device for all-fiber pulsed lasers.

Author Contributions: Conceptualization, P.H. and K.Y.; methodology, J.H.; software, P.H.; validation, J.M., H.N., R.W., B.Z. and T.L.; writing—original draft preparation, P.H.; writing—review and editing, K.Y.; funding acquisition, K.Y. All authors have read and agreed to the published version of the manuscript. 
Funding: This research was funded by National Key Research and Development Program of China (2017YFB0405204), National Natural Science Foundation of China (NSFC) (No. 61775119), Key Research and Development Program of Shandong Province (2017CXCC0808), Shenzhen Science and Technology Research and Development Funds (JCYJ 20180305163932273), and the Program of State Key Laboratory of Quantum Optics and Quantum Optics Devices (KF201908).

Institutional Review Board Statement: Not applicable.

Informed Consent Statement: Not applicable.

Data Availability Statement: Data are contained within this article.

Conflicts of Interest: The authors declare no conflict of interest.

Sample Availability: Samples of the compounds are not available from the authors.

\section{References}

1. Laroche, M.; Chardon, A.M.; Nilsson, J.; Shepherd, D.P.; Clarkson, W.A.; Girard, S.; Moncorge, R. Compact diode-pumped passively Q-switched tunable Er-Yb double-clad fiber laser. Opt. Lett. 2002, 27, 1980-1982. [CrossRef] [PubMed]

2. Richardson, D.J.; Nilsson, J.; Clarkson, W.A. High power fiber lasers: Current status and future perspectives. J. Opt. Soc. Am. B 2010, 27, B63-B92. [CrossRef]

3. Ahmad, H.; Muhammad, F.D.; Chang, H.P.; Thambiratnam, K. Dual-wavelength fiber lasers for the optical generation of microwave and terahertz radiation. IEEE J. Sel. Top. Quant. 2014, 20, 166-173. [CrossRef]

4. Aziz, N.A.; Latiff, A.A.; Lokman, M.Q.; Hanafi, E.; Harun, S.W. Zinc oxide-based Q-switched erbium-doped fiber laser. Chin. Phys. Lett. 2017, 34, 43-45. [CrossRef]

5. Ahmad, H.; Reduan, S.A. Passively Q-switched s-band thulium fluoride fiber laser with multi-walled carbon nanotube. Chin Opt. Lett. 2018, 16, 010609-1-010609-5. [CrossRef]

6. Rosdin, R.Z.R.R.; Ahmad, F.; Ali, N.M.; Harun, S.W.; Arof, H. Q-switched Er-doped fiber laser with low pumping threshold using graphene saturable absorber. Chin. Opt. Lett. 2014, 12, 091404. [CrossRef]

7. Dong, X.; Tian, J.; Yu, Z.; Song, Y. Q-switched Er-doped fiber laser with single-walled carbon nanotube saturable absorber by evanescent field. Chin. Opt. Lett. 2014, 12, S21402. [CrossRef]

8. Ahmad, H.; Aidit, S.N.; Ooi, S.I.; Tiu, Z.C. Tunable passively Q-switched ytterbium-doped fiber laser with mechanically exfoliated gase saturable absorber. Chin. Opt. Lett. 2018, 16, 020014. [CrossRef]

9. Liu, M.; Liu, W.; Yan, P.; Fang, S.; Teng, H.; Wei, Z. High-power MoTe 2 -based passively Q-switched erbium-doped fiber laser. Chin. Opt. Lett. 2018, 16, 020007.

10. Novoselov, K.S.; Geim, A.K.; Morozov, S.V.; Jiang, D.; Katsnelson, M.I.; Grigorieva, I.V.; Dubonos, S.V.; Firsov, A.A. Twodimensional gas of massless Dirac fermions in graphene. Nature 2005, 438, 197-200. [CrossRef]

11. Zhang, Y.B.; Tan, Y.W.; Stormer, H.L.; Kim, P. Experimental observation of the quantum Hall effect and Berry's phase in graphene. Nature 2005, 438, 201-204. [CrossRef]

12. Mak, K.F.; Lee, C.; Hone, J.; Shan, J.; Heinz, T.F. Atomically thin $\mathrm{MoS}_{2}$ : A new direct-gap semiconductor. Phys. Rev. Lett. 2010, 105, 136805.1-136805.4. [CrossRef] [PubMed]

13. Splendiani, A.; Liang, S.; Zhang, Y.B.; Li, T.S.; Kim, J.; Chim, C.Y.; Galli, G.; Wang, F. Emerging photoluminescence in monolayer $\mathrm{MoS}_{2}$. Nano Lett. 2010, 10, 1271-1275. [CrossRef] [PubMed]

14. Lu, S.; Du, L.; Kang, Z.; Li, J.; Huang, B.; Jiang, G. Stable dissipative soliton generation from Yb-doped fiber laser modulated via evanescent field interaction with gold nanorods. IEEE Photon. J. 2018, 10, 1-8. [CrossRef]

15. Khaz Ae Inezhad, R.; Kassani, S.H.; Paulson, B.; Jeong, H.; Gwak, J.; Rotermund, F. Ultrafast nonlinear optical properties of thin-solid DNA film and their application as a saturable absorber in femtosecond mode-locked fiber laser. Sci. Rep. 2017, 7, 41480. [CrossRef] [PubMed]

16. Kou, L.; Frauenheim, T.; Chen, C. Nanoscale multilayer transition-metal dichalcogenide heterostructures: Band gap modulation by interfacial strain and spontaneous polarization. J. Phys. Chem. Lett. 2013, 4, 1730-1736. [CrossRef] [PubMed]

17. Yin, Z.; Li, H.; Li, H.; Jiang, L.; Shi, Y.; Sun, Y.; Lu, G.; Zhang, Q.; Chen, X.; Zhang, H. Single-layer MoS 2 phototransistors. ACS Nano 2012, 6, 74-80. [CrossRef] [PubMed]

18. Huang, Z.; Zhang, W.; Zhang, W. Computational search for two dimensional $\mathrm{MX}_{2}$ semiconductors with possible high electron mobility at room temperature. Materials 2016, 9, 716. [CrossRef]

19. Xia, H.D.; Li, H.P.; Lan, C.Y.; Li, C.; Zhang, X.X.; Zhang, S.J.; Liu, Y. Ultrafast erbium-doped fiber laser mode-locked by a CVD-grown molybdenum disulfide $\left(\mathrm{MoS}_{2}\right)$ saturable absorber. Opt. Express 2014, 22, 17341-17348. [CrossRef]

20. Mao, D.; Wang, Y.; Ma, C.; Han, L.; Jiang, B.; Gan, X.; Hua, S.; Zhang, W.; Mei, T.; Zhao, J. WS 2 mode-locked ultrafast fiber laser. Sci. Rep. 2015, 5, 7965. [CrossRef]

21. Luo, Z.Q.; Li, Y.Y.; Zhong, M.; Huang, Y.Z.; Wan, X.J.; Peng, J.; Weng, J. Nonlinear optical absorption of few-layer molybdenum diselenide $\left(\mathrm{MoSe}_{2}\right)$ for passively mode-locked soliton fiber laser. Photonics Res. 2015, 3, A79-A86. [CrossRef] 
22. Mao, D.; She, X.Y.; Du, B.B.; Yang, D.X.; Zhang, W.D.; Song, K.; Cui, X.Q.; Jiang, B.Q.; Peng, T.; Zhao, J.L. Erbium-doped fiber laser passively mode locked with few-layer $\mathrm{WSe}_{2} / \mathrm{MoSe}_{2}$ nanosheets. Sci. Rep. 2016, 6, 23583. [CrossRef] [PubMed]

23. Zhang, M.; Hu, G.; Hu, G.; Howe, R.; Chen, L.; Zheng, Z. Yb- and Er-doped fiber laser Q-switched with an optically uniform, broadband $\mathrm{WS}_{2}$ saturable absorber. Sci. Rep. 2015, 5, 11453-11461. [CrossRef] [PubMed]

24. Patel, K.; Solanki, G.K.; Patel, K.D.; Pataniya, P.; Tannarana, M.; Chauhan, P. Optoelectronic devices based on chemical vapour transport grown $\mathrm{NbSe}_{2}$ crystals. AIP Conf. Proc. 2019, 2100, 020130-1-020130-4.

25. Le, Q.V.; Choi, J.Y.; Kim, S.Y. Recent advances in the application of two-dimensional materials as charge transport layers in organic and perovskite solar cells. Flatchem 2017, 2, 1-13.

26. Bachmann, R.; Kirsch, H.C.; Geballe, T.H. Optical properties and superconductivity of NbSe 2 . Solid State Commun. 1971, 9, 57-60. [CrossRef]

27. Shi, Y.; Li, W.; Lu, W. Passively Q-switched Er-doped fiber laser based on $\mathrm{NbSe}_{2}$ quantum dot saturable absorber. In Proceedings of the Asia Communications and Photonics Conference (ACP), Hangzhou, China, 26-29 October 2018.

28. Shi, Y.H.; Long, H.; Liu, S.X.; Tsang, Y.H.; Wen, Q. Ultrasmall $2 \mathrm{D} \mathrm{NbSe} 2$ based quantum dots used for low threshold ultrafast lasers. J. Mater. Chem. C 2018, 6, 12638-12642. [CrossRef]

29. Yang, H. Niobium diselenide nanosheets for a vector soliton fiber laser. J. Mater. Chem. C 2020, 8, 14954-14958. [CrossRef]

30. Chen, L.L.; Du, L.; Li, J.; Yang, L.L.; Yi, Q.; Zhao, C.J. Dissipative soliton generation from Yb-doped fiber laser modulated by mechanically exfoliated $\mathrm{NbSe}_{2}$. Front. Phys. 2020, 8, 1-7. [CrossRef]

31. Wang, H.; Huang, X.; Lin, J.; Cui, J.; Chen, Y.; Zhu, C. High-quality monolayer superconductor NbSe 2 grown by chemical vapour deposition. Nat. Commun. 2017, 8, 394. [CrossRef]

32. Chen, B.H.; Zhang, X.Y.; Wu, K.; Wang, H.; Wang, J.; Chen, J.P. Q-switched fiber laser based on transition metal dichalcogenides $\mathrm{MoS}_{2}, \mathrm{MoSe}_{2}, \mathrm{WS}_{2}$, and WSe 2 . Opt. Express 2015, 23, 26723. [CrossRef] [PubMed]

33. Wang, J.; Luo, Z.; Zhou, M.; Ye, C.; Fu, H.; Cai, Z.; Cheng, H.; Xu, H.; Qi, W. Evanescent-light deposition of graphene onto tapered fibers for passive Q-Switch and mode-locker. IEEE Photon. J. 2012, 4, 1295-1305. [CrossRef]

34. Li, L.; Lv, R.D.; Liu, S.C.; Wang, Y.G.; Chen, Z.D.; Wang, J. Transition metal dichalcogenide $\left(\mathrm{WS}_{2}\right.$ and $\left.\mathrm{MoS}_{2}\right)$ saturable absorbers for Q-switched Er-doped fiber lasers. Laser Phys. 2018, 28, 055106. [CrossRef]

35. Guo, L.; Wen, J.G. Numerically Analysis on the Role of the Modulation Depth in an All-Fiber Erbium-Doped Laser with Normal Cavity Dispersion. Appl. Mech. Mater. 2013, 411-414, 761-764. [CrossRef]

36. Xu, X.; Jiang, M.; Li, D.; Wang, R.D.; Ren, Z.Y.; Bai, J.T. Passive Q-switching based on ReS $_{2}$ saturable absorber in Er-doped fiber laser at $1532 \mathrm{~nm}$. Opt. Quant. Electron. 2018, 50, 39. [CrossRef]

37. Liu, S.C.; Zhang, Y.N.; Li, L.; Wang, Y.G.; Lv, R.D.; Wang, X.; Chen, Z.D.; Wei, L.L. Er-doped Q-switched fiber laser with a black phosphorus / polymethyl methacrylate saturable absorber. Appl. Opt. 2018, 57, 1292-1295. [CrossRef]

38. Hu, P.; Liu, Y.; Guo, L.P.; Ge, X.L.; Liu, X.J.; Yu, L.J.; Liu, Q.H. Passively Q-switched erbium-doped fiber laser based on antimonene as saturable absorber. Appl. Opt. 2019, 58, 7845. [CrossRef] 